\title{
Aromatase Inhibitors and Contralateral Breast Cancer in BRCA Mutation Carriers: A long-term Follow-up
}

Maryam Nemati Shafaee ( $\nabla$ shafaee@bcm.edu )

Baylor College of Medicine https://orcid.org/0000-0001-8478-5340

Kristina Goutsouliak

Baylor College of Medicine

Heather Lin

The University of Texas MD Anderson Cancer Center

Therese B Bevers

The University of Texas MD Anderson Cancer Center

Angelica Gutierrez-Barrera

The University of Texas MD Anderson Cancer Center

Melissa Bondy

Stanford Cancer Institute

Banu Arun

The University of Texas MD Anderson Cancer Center

\section{Research Article}

Keywords: BRCA Mutation Carriers, Contralateral Breast Cancer(CBC), Aromatase, Follow-up, breast cancer (BC)

Posted Date: December 20th, 2021

DOI: https://doi.org/10.21203/rs.3.rs-1158051/v1

License: (c) (i) This work is licensed under a Creative Commons Attribution 4.0 International License. Read Full License 


\section{Abstract}

Background: Deleterious BRCA mutations confer a significant lifetime risk of breast cancer (BC) as well as contralateral $\mathrm{BC}(\mathrm{CBC})$ in patients who do not undergo prophylactic mastectomy. Prior reports have suggested that tamoxifen reduces the risk of $\mathrm{CBC}$ in BRCA mutation carriers. Whether aromatase inhibitors (Al) have the same effect is unknown.

Methods: This is a retrospective review of patients diagnosed with non-metastatic ER+ BC between 20042014 with known BRCA mutation status. Patients were followed from primary diagnosis until CBC diagnosis or death. Median follow up was 11.5 years. Risk of CBC was evaluated as time to event.

Results: 935 subjects were included in this analysis, with 53 BRCA1 mutation carriers, and 94 BRCA2 mutation carriers. Median age at diagnosis was 42.7 years. Seventy-two percent (676) received tamoxifen and $43 \%$ (405) received Al. A total of 66 CBCs occurred, of which 10\% (15/147) occurred in BRCA mutation carriers vs \%6.5 (51/788) in BRCA wild type. Multivariate analyses indicated that BRCA status and $\mathrm{Al}$ use were significantly associated with $\mathrm{CBC}$ risk. Al use resulted in a significant reduction in risk of $\mathrm{CBC}(\mathrm{HR} 0.44, \mathrm{p}=0.004)$ regardless of the BRCA mutation status. Tamoxifen use was not associated with reduced risk of $\mathrm{CBC}$.

Conclusions: This is the first report showing that Als reduce the risk of $C B C$ in BRCA mutation carriers. The potential role of Als as chemoprevention should be validated in larger independent cohorts.

\section{Introduction}

BRCA $1 \frac{1}{2}$ mutations carriers have more than $70 \%$ risk of breast cancer $(B C)$ by age 80 [1-3]. BRCA1 mutation associated $\mathrm{BCs}$ are predominantly high grade triple negative cancers, while BRCA2 associated $B C$ s often resemble the general $B C$ subtype distribution and are frequently ER positive [4]. The risk of contralateral $\mathrm{BC}(\mathrm{CBC})$ in BRCA mutation carriers with personal history of $\mathrm{BC}$ is about $3 \%$ per year [5], with 10 year cumulative risk of about 35\% [5-7]. Risk of $C B C$ seems to be higher in BRCA1 mutations carriers compared to BRCA2, but also increases with higher number of affected first and second-degree relatives [8]. Bilateral prophylactic mastectomy is an effective intervention for risk reduction in BRCA mutation carriers with resultant $90-95 \%$ lower risk of BC [9]. Current NCCN guidelines recommend bilateral mastectomy in BRCA mutation carriers who are younger or equal to 35 years of age or premenopausal [10]. Bilateral salpingo-oophorectomy (BSO) which reduces the risk of ovarian cancer, also reduces the risk of $\mathrm{BC}$ in this patient population [11]. For patients affected with $\mathrm{BC}$, prophylactic mastectomy for $\mathrm{CBC}$ risk reduction is recommended [10].

For BRCA mutation carriers who choose not to undergo risk reducing mastectomy, yearly screening with mammogram and MRI is recommended [12]. Chemoprevention in this patient population is a debated topic, with highly variable recommendation guidelines $[4,13]$. 
Currently there are no prospective data on tamoxifen use as a chemo-preventive agent for primary risk reduction in BRCA mutation carriers, however, in a recent meta-analysis of the literature, Xu and colleagues report a significant $44 \%$ risk reduction in both BRCA1 and BRCA2 mutation carriers for development of second $B C$ [14]. To date there are no data on adjuvant aromatase inhibitors (AI) and risk of $\mathrm{CBC}$ in BRCA mutation carriers. Given supportive data for Al use and $\mathrm{CBC}$ risk reduction in women with primary $B C$ [15], we sought to investigate whether Als have any roles in reducing the risk of $C B C$ in $B R C A$ mutation carriers. We evaluated data from MD Anderson Cancer BC patients who had a personal history of hormone receptor positive $\mathrm{BC}$, and known BRCA mutation status.

\section{Methods}

\section{Study Population and Data Collection}

Women diagnosed with stages I-III, who underwent genetic counseling and testing for BRCA between year 2004 and 2014 were identified from a prospectively maintained High Risk Genetics research registry at UT MD Anderson Cancer Center. Patients whose primary BC was ER-positive and who never underwent contralateral prophylactic mastectomy during the follow up period were included in the analysis. We excluded women diagnosed with metastatic disease at initial presentation or within 1 year from diagnosis of primary BC, as well as women diagnosed with a gynecologic malignancy during the followup period. The study was IRB approved at MD Anderson Cancer center. We abstracted age at diagnosis, menopausal status, stage, demographics, tumor markers, use of tamoxifen, anastrozole, letrozole, exemestane, goserelin, and Lupron. For each hormonal therapy the start date and discontinuation dates were recorded. Patients who had less than 6 months of endocrine therapy were considered endocrine therapy naïve. History of bilateral salpingo-oophorectomy (BSO), the date of CBC diagnosis and BRCA mutation status (e.g. 1 vs. 2 vs. negative) were collected. Each episode of $C B C$ was counted as a single event. Pathology details were collected from pathology reports, and the date of last follow-up or death was recorded.

\section{Exposure and Outcome Assessment}

Endocrine treatment including Tamoxifen and of the three Als, alone or sequentially for at least 6 months without interruption was considered as the exposure. Outcome was development of $\mathrm{CBC}$ during the follow up period. Cox regression analysis was performed to evaluate whether $\mathrm{CBC}$ affects overall survival (OS).

\section{Statistical Analysis}

The student t-test/Wilcoxon test and ANOVA/Kruskal-Wallis tests were used to compare continuous variables between different patient groups. The chi-square test or the Fisher's exact test were applied to assess the association between two categorical variables. Risk of $\mathrm{CBC}$ was evaluated as time to event, defined as the time interval from the diagnosis of primary $B C$ to time of $C B C$ diagnosis (TTCBC). 
Cumulative incidence rate of $\mathrm{CBC}$ was calculated in the presence of death as a competing risk. The effects of covariates on the cumulative incidence function of $\mathrm{CBC}$ were evaluated in the univariate setting using Gray's test. In the multivariate setting, Fine and Gray's method was used to model the probability sub-distribution function of $\mathrm{CBC}$ by applying decreasing weights to patients who died before experiencing $\mathrm{CBC}[3,4]$. A multivariate model was obtained by first including a set of candidate predictor variables including BRCA mutation status, adjuvant tamoxifen and/or Al with a p-value $<0.10$ in the univariate analysis. A backward elimination was then performed using 0.05 for the significance level of the Wald chi-square for an effect to stay in the model. Once the list of variables to be used in our final model was selected, we further assessed the interaction effect on TTCBC between adjuvant Al or tamoxifen therapy and BRCA mutation status. The purpose of the interaction analyses was to evaluate whether or not the magnitude or direction of the association between adjuvant Al or tamoxifen therapy and TTCBC differed by BRCA status. Two-sided $p$ value $<0.05$ were considered statistically significant. Univariate Cox proportional hazards regression was performed regression to estimate the hazard ratio (HR) associated with risk of $\mathrm{CBC}$ and death at 5 and 10 years post primary diagnosis in a landmark analysis. SAS version 9.4 and S-Plus version 8.04 is used to carry out the computations for all analyses.

\section{Results}

A total of 1,083 patients met the inclusion criteria. After applying the exclusion criteria, 935 women were included in the analysis. Fifthy three patients had a BRCA1 pathogenic variant and 94 had BRCA2 pathogenic variant. 788 did not have a deleterious BRCA mutation, (Table 1). Median age at diagnosis of primary BC was 42.7. seventy two percent of the patients were White, $15.5 \%$ Hispanic, 6.5 Black, $4.6 \%$ Asian and $1.2 \%$ other racial categories. Four hundred fifty-five (49\%) of our patients received only tamoxifen as adjuvant therapy, $405(43.3 \%)$ received $\mathrm{Al}$ or a combination of tamoxifen plus an $\mathrm{Al}$, and 75 patients $(8 \%)$ did not receive any endocrine therapy. Median follow up time was 11.5 years with date of last database inquiry for follow up on 12/3/2020. BRCA1 mutation carriers were more frequently diagnosed with grade 3 disease compared to BRCA wild type and BRCA2 mutation carriers (79\% vs. $41 \%$ ). BRCA mutation carriers were less frequently diagnosed with Her2 positive disease compared to BRCA1 and BRCA wild type patients ( $5 \%$ vs. $13.3 \%$ vs. $16.4 \%$ ). BRCA mutation carriers had more frequently underwent BSO compared to BRCA wild type. Total of 66 patients developed $C B C$, where 51 occurred in BRCA wild type patients, 3 among BRCA1 carriers and 12 among BRCA2 carriers.

Table 1. Patient characteristics 


\begin{tabular}{|c|c|c|c|}
\hline \multirow[b]{2}{*}{ Patient variable } & \multirow[b]{2}{*}{$\begin{array}{l}\text { BRCA Wild-type } \\
\quad \mathrm{N}=789\end{array}$} & \\
\hline & & $\begin{array}{c}\mathrm{BRCA} 1 \\
\mathrm{~N}=53\end{array}$ & $\begin{array}{c}\text { BRCA } 2 \\
\mathrm{~N}=94\end{array}$ \\
\hline \multicolumn{4}{|c|}{ Age at Diagnosis of first BC (yrs) } \\
\hline$\leq 50$ & $626(79 \%)$ & $45(85 \%)$ & $76(81 \%)$ \\
\hline$>50$ & $162(21 \%)$ & $8(15 \%)$ & $18(19 \%)$ \\
\hline Median & 43.4 & 40.7 & 43.7 \\
\hline \multicolumn{4}{|l|}{ Nuclear Grade } \\
\hline Grade 1 & $63(8 \%)$ & $2(4 \%)$ & $1(1 \%)$ \\
\hline Grade 2 & $381(48 \%)$ & $8(15 \%)$ & $50(53 \%)$ \\
\hline Grade 3 & $323(41 \%)$ & $42(79 \%)$ & $39(41 \%)$ \\
\hline \multicolumn{4}{|c|}{ Hormone receptor status (\%) } \\
\hline$P R$ positive & $633(80 \%)$ & $29(55 \%)$ & $74(79 \%)$ \\
\hline \multicolumn{4}{|l|}{ Her 2 Status (\%) } \\
\hline Her 2 positive & $113(16.4 \%)$ & $6(13.3 \%)$ & $4(5 \%)$ \\
\hline Her 2 negative & $575(83.6 \%)$ & $39(86.7 \%)$ & $76(95 \%)$ \\
\hline \multicolumn{4}{|l|}{ Stage } \\
\hline I & $249(31.6 \%)$ & $14(26.4 \%)$ & $25(26.6 \%)$ \\
\hline II & $378(48 \%)$ & $28(52.8 \%)$ & $50(53.2 \%)$ \\
\hline III & $161(20.4 \%)$ & $11(20.8 \%)$ & $19(20.2 \%)$ \\
\hline \multicolumn{4}{|c|}{ Adjuvant Hormonal Therapy (\%) } \\
\hline Tamoxifen & $407(52 \%)$ & $16(30 \%)$ & $32(34 \%)$ \\
\hline$A I$ & $145(18 \%)$ & $14(26 \%)$ & $25(27 \%)$ \\
\hline$A I+$ Tamoxifen & $176(22 \%)$ & $14(26 \%)$ & $31(33 \%)$ \\
\hline None & $60(8 \%)$ & $9(17 \%)$ & $6(6 \%)$ \\
\hline \multicolumn{4}{|l|}{$\mathrm{BSO}$} \\
\hline yes & $145(18.5 \%)$ & $30(57 \%)$ & $51(54 \%)$ \\
\hline no & $640(81.5 \%)$ & $23(43 \%)$ & $43(46 \%)$ \\
\hline $\mathrm{CBC}$ & $51(6.5 \%)$ & $3(6 \%)$ & $12(12.8 \%)$ \\
\hline
\end{tabular}

Abbreviations: BC, Breast Cancer; ER, Estrogen receptor; CBC, contralateral breast cancer; Al, Aromatase Inhibitor; BSO, Bilateral Salpingo-oophorectomy; Her, Herceptin

In the univariate analysis (Table 2.), Al use in sequence with Tamoxifen or alone was associated with reduced risk of $\mathrm{CBC}$, hazard ratio (HR) 0.46 (95\% Cl 0.26-0.79) (Figure 1). Compared to no hormone therapy usage, tamoxifen alone without Al therapy was not associated with reduced risk of CBC: HR 0.65 (95\% $\mathrm{Cl}$ 0.35-1.31) (Figure 2). Al use alone compared to no endocrine therapy use was also associated with lower risk of $\mathrm{CBC}$ with $\mathrm{HR} 0.4$ (95\% $\mathrm{Cl} 0.16-0.99)$ (Figure 3). Having a BRCA mutation was associated with an increased risk of $\mathrm{CBC}$ : HR 1.75 (95\% $\mathrm{Cl} 0.99$ - 3.1), Figure 4). Stage or age at diagnosis of primary $\mathrm{BC}$, tumor grade, and history of BSO were not significantly associated with risk of CBC.

Table 2. Risk of Contralateral Breast Cancer associated with selected factors 


\begin{tabular}{|c|c|c|c|c|c|c|}
\hline \multicolumn{4}{|c|}{ Univariate Analysis } & \multicolumn{3}{|c|}{ Multivariate Analysis } \\
\hline Factor & & HR & $95 \% \mathrm{Cl}$ & HR & $95 \% \mathrm{Cl}$ & $\mathbf{P}$ \\
\hline Age at Diagnosis & Per Year Increase & 1.01 & $0.98-1.03$ & & & \\
\hline BSO & Yes vs No & 1.00 & $0.58-1.7$ & & & \\
\hline BRCA + & BRCA Carrier vs. Non-Carrier & 1.75 & 0.99-3.1 & & & \\
\hline BRCA 1 & BRCA 1 vs. Non-Carrier & 1.13 & 0.34-3.72 & & & \\
\hline BRCA 2 & BRCA 2 vs. Non-Carrier & 2.03 & $1.1-3.7$ & 2.2 & $1.2-4.44$ & 0.048 \\
\hline \multirow[t]{2}{*}{ Stage At Diagnosis } & Stage II vs. I & 0.75 & $0.45-1.26$ & & & \\
\hline & Stage III vs. 1 & 0.5 & $0.23-1.01$ & & & \\
\hline \multirow[t]{2}{*}{ Nuclear Grade } & G2 vs G1 & 0.57 & $0.26-1.27$ & & & \\
\hline & G3 vs G1 & 0.46 & $0.2-1.06$ & & & \\
\hline Tamoxifen & Yes vs No & 0.88 & $0.52-1.5$ & & & \\
\hline Al & Yes vs No & 0.46 & $0.26-0.79$ & 0.44 & $0.25-0.77$ & 0.004 \\
\hline \multirow[t]{3}{*}{ Tamoxifen / Al } & Al alone vs None & 0.4 & $0.16-0.99$ & 0.39 & $0.16-0.97$ & 0.017 \\
\hline & Tamoxifen alone & 0.65 & $0.35-1.31$ & & & \\
\hline & Tamoxifen / Al & 0.26 & $0.1-0.66$ & 0.26 & $0.1-0.66$ & 0.017 \\
\hline
\end{tabular}

In multivariate analysis (Table 2.), Al use remained significantly associated with lower risk of CBC: HR 0.44 (95\% Cl 0.25-0.77). BRCA 2 was associated with increased risk of CBC - HR 2.2 (95\% Cl 1.2-4.44) (Figure 5.). Use of Al was associated with reduced the risk of $\mathrm{CBC}$ regardless of the BRCA status, whether alone HR0.39 (95\% Cl 0.16-0.97) or sequentially with tamoxifen HR 0.26 (95\% Cl 0.1-0.66).

On evaluating whether there were any interactions between Al therapy and BRCA status, we found no significant interaction with Wald Chi-square coefficient of 1.3 and $p=0.25$. Similarly we found no significant interaction between BRCA status and Tamoxifen $(p=0.75)$.

Contralateral $\mathrm{BC}$ at 5 or 10 years post primary diagnosis were not significantly associated with increased hazards of death (Figures 6 and 7). Overall survival post primary diagnosis was not different between BRCA mutation carriers versus noncarriers (Figure 8).

\section{Discussion}

Our results suggest that after diagnosis of primary $\mathrm{BC}$, in our overall cohort, adjuvant Al therapy alone or used sequentially with tamoxifen reduces risk of $\mathrm{CBC}$. This risk reduction is irrespective of the BRCA 
mutation pattern.

Aromatase inhibitor therapy has a recognized profile for reducing risk of CBC. In MA. 17R, a phase 3, randomized, double-blind, placebo-controlled trial involving postmenopausal women with primary $\mathrm{BC}$ who had received 4.5 to 6 years of adjuvant hormone therapy, extending adjuvant Al therapy with letrozole to 10 y resulted in lower risk of $\mathrm{CBC}, \mathrm{HR} 0.42$ with $\mathrm{P}=0.007$, compared to placebo, which translates to a $34 \%$ lower incidence of CBC. Continuing this therapy was nonetheless associated with more bone related side effects including osteoporosis [16]. In the 10-year analysis of the ATAC trial (Arimidex, Tamoxifen, Alone or in Combination) which randomized post-menopausal women with early stage invasive $\mathrm{BC}$ to anastrozole or tamoxifen or combination of the two, anastrozole use resulted in persistently lower risk of $\mathrm{CBC}$ compared to tamoxifen regardless of hormone receptor status of the primary cancer, HR 0.68 (95\% Cl 0.50 to 0.91) [17]. Given the findings in our report, the benefit of Al therapy for reducing the risk of CBC appears to extend to BRCA mutation carriers, and there may be biological plausibility to this result.

BRCA gene mutations, specifically BRCA1, frequently predispose to development of hormone receptor negative, or triple negative BCs [18]. Between 4-27\% of BCs in BRCA1 mutation carriers express estrogen receptor. This number is higher in BRCA2 mutation carriers where up to $65 \%$ are reported to have ER expression $[18,19]$. Data on potential interaction between estrogen, estrogen receptor, and BRCA genes are mounting although limited at this time. There is preclinical evidence for interaction between BRCA1 gene and one of the two main kinds of estrogen receptor, ER alpha $(E R \propto)$ which is activated by circulating estrogen. Wild-type BRCA1 is found to inhibit tumorigenesis by inhibiting ER $\propto$ induced VEGF gene transcription [20]. BRCA1 mutation carriers who are $\geq 50$ years at diagnosis of their first invasive BC have a higher likelihood of having an ER+ cancer compared to those younger than age 50,57\% vs 29\% [21]. Additionally, these ER+ tumors have an "intermediate" pathological phenotype, with features of both ER+ sporadic BCs as well as ER negative (ER-) rapidly proliferating BRCA associated tumors [21].

Tumor genomic profiling shows loss of the wild-type BRCA1 allele in up to $83 \%$ of ER+ BRCA1 breast tumors [22]. On microarray molecular profiling, BRCA2 associated BCs are more likely to be high grade and ER+, exhibiting a luminal phenotype without a specific pattern for frequently altered proteins, e.g. p53, bcl2 and cyclin D1 [23]. Different cells of origin, i.e. early progenitor versus cancer stem cells, have been proposed to be the underlying explanation for ER+ vs ER+ tumor types observed in BRCA mutated BCs [24]. Prophylactic oophorectomy in BRCA mutation carriers reduces the risk of BC [11], and there are reports for tamoxifen acting as a chemo-preventive agent for reducing rate of $\mathrm{CBC}$ in this population (Table 3.). All of this is suggestive of a role for hormone therapy for primary prevention, in BRCA mutation carriers. 
Table 3

Tamoxifen as Risk Reducer of CBC in BRCA 1/2 Mutation Carriers

\begin{tabular}{|c|c|c|c|c|c|}
\hline $\begin{array}{l}\text { Author, } \\
\text { year }\end{array}$ & Study Design & $\begin{array}{l}\text { Number of BRCA } \\
\text { mutation carriers } \\
\text { n (\%) }\end{array}$ & $\begin{array}{l}\text { Follow- } \\
\text { up (y) }\end{array}$ & $\begin{array}{l}\text { Adjuvant } \\
\text { Tamoxifen } \\
\text { Use }\end{array}$ & CBC Risk \\
\hline \multirow{3}{*}{$\begin{array}{l}\text { Metcalfe, } \\
\text { et al. } 2004 \\
\text { [5] }\end{array}$} & \multirow{3}{*}{$\begin{array}{l}\text { Retrospective } \\
\text { Cohort }\end{array}$} & & \multirow{3}{*}{$\begin{array}{l}\text { Mean } \\
9.2\end{array}$} & \multirow{3}{*}{$\begin{array}{l}30 \% \\
\text { overall }\end{array}$} & \multirow{3}{*}{$\begin{array}{l}\mathrm{HR}^{\star} 0.59(95 \% \mathrm{Cl} 0.26 \text { to } \\
1.33)\end{array}$} \\
\hline & & $\begin{array}{l}327 \text { BRCA1 } \\
(66.6 \%)\end{array}$ & & & \\
\hline & & $\begin{array}{l}152 \text { BRCA } 2 \\
(31 \%)\end{array}$ & & & \\
\hline \multirow{3}{*}{$\begin{array}{l}\text { Reading et } \\
\text { al. 2010 } \\
{[25]}\end{array}$} & \multirow[t]{3}{*}{ Case-control } & 181 & \multirow[t]{3}{*}{ N/A } & \multirow{3}{*}{$\begin{array}{l}11.3 \% \text { in } \\
\text { BRCA1 } \\
47 \% \text { in } \\
\text { BRCA2 }\end{array}$} & \multirow{2}{*}{$\begin{array}{l}\mathrm{RR}^{\star \star} \text { in mutation } \\
\text { carriers } 0.7(95 \% \mathrm{Cl} 0.3 \\
-1.8)\end{array}$} \\
\hline & & & & & \\
\hline & & 72 BRCA 2 (40\%) & & & $\begin{array}{l}\text { RR in mutation non- } \\
\text { carriers } 0.7(95 \% \mathrm{Cl} \text { : } 0.6- \\
1.0) ; \text { P-value }=0.03\end{array}$ \\
\hline \multirow{4}{*}{$\begin{array}{l}\text { Philips et } \\
\text { al. 2013 } \\
\text { [26] }\end{array}$} & \multirow{4}{*}{$\begin{array}{l}\text { Retrospective } \\
\text { Cohort }\end{array}$} & 2,464 & \multirow{4}{*}{$\begin{array}{l}\text { Median } \\
6.6\end{array}$} & \multirow{4}{*}{$\begin{array}{l}24 \% \text { in } \\
\text { BRCA } 1 \\
52 \% \text { in } \\
\text { BRCA2 }\end{array}$} & BRCA1 \\
\hline & & $\begin{array}{l}1,583 \text { BRCA1 } \\
(64 \%)\end{array}$ & & & $\begin{array}{l}\text { HR } 0.38(95 \% \mathrm{Cl}, 0.27 \text { to } \\
0.55)\end{array}$ \\
\hline & & 881 BRCA 2 & & & BRCA2 \\
\hline & & & & & $\begin{array}{l}\text { HR } 0.33(95 \% \mathrm{Cl}, 0.22 \text { to } \\
0.50)\end{array}$ \\
\hline \multirow{7}{*}{$\begin{array}{l}\text { Gronwald } \\
\text { et al. } 2014 \\
\text { [27] }\end{array}$} & \multirow[t]{7}{*}{ Case-control } & 1,505 & \multirow[t]{7}{*}{$\mathrm{N} / \mathrm{A}$} & \multirow{7}{*}{$\begin{array}{l}22 \% \\
\text { overall }\end{array}$} & Up to 1 y of Tamoxifen \\
\hline & & $\begin{array}{l}1,256 \text { BRCA } 1 \\
(83.5 \%)\end{array}$ & & & OR 0.37 (95\% Cl: 0.20 \\
\hline & & \multirow{5}{*}{$\begin{array}{l}248 \mathrm{BRCA} 2 \\
(16.5 \%)\end{array}$} & & & $-0.69)$ \\
\hline & & & & & 1-4 y of Tamoxifen \\
\hline & & & & & $\begin{array}{l}\text { OR } 0.53 \text { (95\% Cl: } 0.32- \\
0.87)\end{array}$ \\
\hline & & & & & $>4 y$ of Tamoxifen \\
\hline & & & & & 0.83 (95\% Cl: $0.44-1.55)$ \\
\hline \multicolumn{6}{|c|}{ * HR: Hazard Ratio } \\
\hline \multicolumn{6}{|c|}{ ** RR: Relative Risk } \\
\hline \$ OR: Odds & & & & & \\
\hline
\end{tabular}

Our results did not find a statistically significant improvement in CBC with tamoxifen use for either BRCA mutation carriers or non-carriers. These results are not aligned with conclusion of a recent meta-analysis 
by Xu et al. [14] which aimed at assessing the effect of tamoxifen use on CBC risk in BRCA1/2 mutation carriers. The studies included in this meta-analysis are summarized in Table 3. In the study by Metcalf [5], 491 women with BRCA1/2 mutations, and stage I or II BC were followed for a median of 9.2 years. During this time, 155 patients underwent prophylactic contralateral mastectomy, of the remaining 336 patients, 97 developed CBCs. Of the 97 episodes of $\mathrm{CBC}, 30 \%$ were ER+ disease. 10-year risk of CBC was reported at $29.5 \%(95 \% \mathrm{Cl}, 20.6-38.3 \%)$. Tamoxifen use was reported in overall $30 \%$ of the patients included in this study. In their adjusted analysis, tamoxifen use was not associated with reduced risk of CBC in BRCA1/2 mutation carriers. Oophorectomy for women younger than age 50 at time of primary BC was protective from CBC. Chemotherapy or radiation were not associated with lower risk of CBC.

A case-control study by Redding et al. [25], the Women's Environment, Cancer, and Radiation Epidemiology (WECARE), included women with $\mathrm{CBC}$ as cases and those with unilateral $\mathrm{BC}$ as controls. In a total of 2197 study subjects 181 were BRCA mutation carriers, $60 \%$ had BRCA1 mutation, and $40 \%$ had BRCA 2 mutation. Two BRCA1 carriers and 12 BRCA2 mutation carriers developed CBC. Adjuvant tamoxifen was not associated with statistically significant lower risk of CBC in BRCA mutation carriers, while it was found to be effective in mutation non-carriers with relative risk of $0.7, p$-value 0.03 .

Phillips et al. [26] performed a retrospective analysis of 2,464 BRCA mutation carriers, where 1,583 had BRCA1 and 881 had BRCA2 mutations. Of these patients, $24 \%$ of the BRCA1 mutation carriers and $52 \%$ of BRCA 2 mutation carriers took adjuvant tamoxifen. Total 520 episodes of CBC were found with median follow-up of the study at 6.6 years. Tamoxifen use was associated with a lower risk of CBC in both BRCA1 and 2 mutation carriers. When stratifying based on BSO, tamoxifen remained significantly associated with lower risk of CBC in patients who did not undergo BSO but was no longer significant in those who had undergone the procedure. The analysis did not adjust for chemotherapy use. Estrogen receptor status of the primary $B C$ was missing for $56 \%$ of the patients, however, the hazard ratios (HR) of $\mathrm{CBC}$ did not differ by ER status of the primary $\mathrm{BC}$.

In a case-control study Granwald et al. [27] evaluated 1504 women with BRCA1 or 2 mutation, where 411 women with bilateral BC were counted as cases, and 1093 women with unilateral BC were considered controls. 331 women had used tamoxifen (22\%); among whom 84 (25\%) had completed four or more years of tamoxifen. The remainder were either actively receiving the therapy at the time of evaluation or had stopped it prior to completing 4 years. Women with up to one year of tamoxifen use, had the highest reduction in the odds ratio $(\mathrm{OR})$ of $\mathrm{CBC}, 0.37$ (p-value 0.001 ) compared to no tamoxifen use. Among women with one to four years of tamoxifen use the OR of CBC was 0.53 ( $p$-value 0.01 ). The OR of CBC among women with four or more years of tamoxifen use was not significantly improved compared to no tamoxifen use, $0.83(p=0.55)$. The authors suggested that the next step would be to conduct a placebocontrolled trial of 1 year of tamoxifen in BRCA mutation carriers for primary prevention purposes.

The literature is generally supportive of tamoxifen use in reducing $\mathrm{CBC}$ in all women post diagnosis of $E R+B C[15,28-31]$. A recent retrospective cohort study of $C B C$ evaluated 7541 patients with history of $\mathrm{BC}$ at Kaiser Permanente with median follow up of 6.3 years. The study reported that both tamoxifen and 
Als reduce $\mathrm{CBC}$. The study reports that 4 or more years of adjuvant tamoxifen use will prevent $3 \mathrm{CBCs}$ per 100 women by 10 years from first diagnosis of primary $E R+B C[15,32]$. The authors report that the effect of tamoxifen for reducing the risk of $\mathrm{CBC}$ is dependent on the duration of therapy and diminishes after discontinuation of the drug. Compliance remains an important issue and aromatase inhibitors have a more favorable profile in conferring longer lasting effects with regards to $\mathrm{CBC}$ risk reduction than tamoxifen [15]. Similarly, a Danish case control study of tamoxifen users versus non-users reported a reduced $\mathrm{HR}$ of $\mathrm{CBC}$ among current users of tamoxifen $\mathrm{HR}$ was $0.44(95 \% \mathrm{Cl}, 0.27-0.71)$ while the hazard of $\mathrm{CBC}$ was not significantly reduced among women who had already completed or discontinued tamoxifen [33].

Likely majority of patients had completed the adjuvant tamoxifen therapy during the follow up period, median 11.5 years which raises the possibility that risk reducing effects of tamoxifen may ware off over time. On the other hand, aromatase inhibitors may have a longer duration of effect post discontinuation. Usually patients who receive tamoxifen are younger on average than women who are prescribed Als. Younger women usually have more aggressive pathology and worse prognosis than older women [34], and therefore, tamoxifen alone maybe an inadequate chemopreventive agent.

While BRCA2 mutation was strongly associated with $\mathrm{CBC}$ in this report, BRCA1 mutation was not. With only 3 episodes of CBC in 53 patients with BRCA 1 mutation, we cannot draw any informative conclusions from this finding.

While several studies have evaluated adjuvant tamoxifen use and CBC in BRCA1/2 mutation carriers, and the metanalysis by Xu et al supports the use of tamoxifen for this indication, however optimum duration of therapy is not known. Additionally, whether tamoxifen's benefit extends to other high-risk mutations, e.g. PALB2, ATM, CHEK2 mutation carriers is unknown. Unfortunately, without any prospective trials on use of tamoxifen or Als for chemoprevention in high-risk mutation carriers, the evidence is limited to adjuvant endocrine therapy use and its effect on $\mathrm{CBC}$, which naturally only includes a minority of BRCA mutation carriers - those with hormone receptor positive subtypes. Currently, endocrine therapy is not indicated for treatment of ER negative BC in this population. The optimum duration of Al for CBC risk reduction is unclear, and our findings do not provide conclusive evidence in this regard. However, this study suggests that Als maybe considered an option in patients with BRCA mutation who are keen on avoiding prophylactic mastectomy. Poly-ADP ribose polymerase (PARP) inhibitors have strong anti-tumor activity in BRCA mutation, however their use as chemoprevention is limited due to toxicity, risk of secondary tumors, as well as drug resistance [35]. Other agents that have been proposed as potential chemoprevention for this population pending further studies include the receptor activator of nuclear factor KB ligant (RANKL), denosumab [4]. At this time, there is insufficient data to consider this agent as a valid chemoprevention option.

Future studies are required to validate our results as well as to identify patient populations among BRCA mutation carriers who stand the highest chance of receiving benefit from this approach. 


\section{Declarations}

\section{Funding}

The authors declare that no funds, grants, or other support were received during the preparation of this manuscript.

\section{Competing Interests}

The authors have no relevant financial or non-financial interests to disclose.

\section{Author Contributions}

All authors contributed to the study conception and design. Material preparation, data collection were performed by [Maryam Nemati Shafaee and Angelica Gutierrez], analysis was performed by [Healther Lin, Maryam Nemati Shafaee, Melissa Bondy and Banu Arun]. The first draft of the manuscript was written by [Maryam Nemati Shafaee] and all authors commented on previous versions of the manuscript. All authors read and approved the final manuscript.

\section{Data Availability}

The datasets generated during and/or analyzed during the current study are not publicly available as the database is actively updated and is the list of active and prior patients receiving care at MD Anderson Cancer Center.

\section{Ethics approval}

This study was performed in line with the principles of the Declaration of Helsinki. Approval was granted by the Ethics Committee of UT MD Anderson Cancer Center.

\section{Consent to participate}

Waiver of informed consent was obtained for this retrospective chart review.

\section{References}

1. Ford, D., et al., Genetic heterogeneity and penetrance analysis of the BRCA1 and BRCA2 genes in BC families. The BC Linkage Consortium. Am J Hum Genet, 1998. 62(3): p. 676-89.

2. Metcalfe, K.A. and S.A. Narod, $B C$ prevention in women with a BRCA1 or BRCA2 mutation. Open Med, 2007. 1(3): p. e184-90.

3. Cortesi, L., H.S. Rugo, and C. Jackisch, An Overview of PARP Inhibitors for the Treatment of BC. Target Oncol, 2021. 16(3): p. 255-282.

4. Kotsopoulos, J., Mutations and BC Prevention. Cancers (Basel), 2018. 10(12). 
5. Metcalfe, K., et al., Contralateral BC in BRCA1 and BRCA2 mutation carriers. J Clin Oncol, 2004. 22(12): p. 2328-35.

6. Verhoog, L.C., et al., Contralateral BC risk is influenced by the age at onset in BRCA1-associated BC. Br J Cancer, 2000. 83(3): p. 384-6.

7. Pierce, L.J., et al., Ten-year multi-institutional results of breast-conserving surgery and radiotherapy in BRCA1/2-associated stage I/II BC. J Clin Oncol, 2006. 24(16): p. 2437-43.

8. Kuchenbaecker, K.B., et al., Risks of Breast, Ovarian, and Contralateral BC for BRCA1 and BRCA2 Mutation Carriers. JAMA, 2017. 317(23): p. 2402-2416.

9. Rebbeck, T.R., et al., Bilateral prophylactic mastectomy reduces $B C$ risk in BRCA1 and BRCA2 mutation carriers: the PROSE Study Group. J Clin Oncol, 2004. 22(6): p. 1055-62.

10. NCC Guidelines Version 4.2021 - BC. 2021: NCCN.org.

11. Rebbeck, T.R., et al., BC risk after bilateral prophylactic oophorectomy in BRCA1 mutation carriers. J Natl Cancer Inst, 1999. 91(17): p. 1475-9.

12. Tung, N.M., et al., Management of Hereditary BC: American Society of Clinical Oncology, American Society for Radiation Oncology, and Society of Surgical Oncology Guideline. J Clin Oncol, 2020. 38(18): p. 2080-2106.

13. Campbell, A.M., et al., Chemoprevention in BRCA1 mutation carriers (CIBRAC): protocol for an open allocation crossover feasibility trial assessing mechanisms of chemoprevention with goserelin and anastrozole versus tamoxifen and acceptability of treatment. BMJ Open, 2018. 8(12): p. e023115.

14. Xu, L., et al., Tamoxifen and risk of contralateral $B C$ among women with inherited mutations in BRCA1 and BRCA2: a meta-analysis. BC, 2015. 22(4): p. 327-34.

15. Gierach, G.L., et al., Association of Adjuvant Tamoxifen and Aromatase Inhibitor Therapy With Contralateral BC Risk Among US Women With BC in a General Community Setting. JAMA Oncol, 2016.

16. Goss, P.E., et al., Extending Aromatase-Inhibitor Adjuvant Therapy to 10 Years. N Engl J Med, 2016. 375(3): p. 209-19.

17. Cuzick, J., et al., Effect of anastrozole and tamoxifen as adjuvant treatment for early-stage BC: 10year analysis of the ATAC trial. Lancet Oncol, 2010. 11(12): p. 1135-41.

18. Foulkes, W.D., et al., Estrogen receptor status in BRCA1- and BRCA2-related BC: the influence of age, grade, and histological type. Clin Cancer Res, 2004. 10(6): p. 2029-34.

19. Honrado, E., J. Benitez, and J. Palacios, The pathology of hereditary BC. Hered Cancer Clin Pract, 2004. 2(3): p. 131-8.

20. Kawai, H., et al., Direct interaction between BRCA1 and the estrogen receptor regulates vascular endothelial growth factor (VEGF) transcription and secretion in BC cells. Oncogene, 2002. 21(50): $\mathrm{p}$. 7730-9.

21. Tung, N., et al., Estrogen receptor positive BCs in BRCA1 mutation carriers: clinical risk factors and pathologic features. BC Res, 2010. 12(1): p. R12. 
22. Lips, E.H., et al., BRCA1-Mutated Estrogen Receptor-Positive BC Shows BRCAness, Suggesting Sensitivity to Drugs Targeting Homologous Recombination Deficiency. Clin Cancer Res, 2017. 23(5): p. 1236-1241.

23. Bane, A.L., et al., BRCA2 mutation-associated BCs exhibit a distinguishing phenotype based on morphology and molecular profiles from tissue microarrays. Am J Surg Pathol, 2007. 31(1): p. 121-8.

24. Lakhani, S.R., K.K. Khanna, and G. Chenevix-Trench, Are estrogen receptor-positive BCs in BRCA1 mutation carriers sporadic? BC Res, 2010. 12(2): p. 104.

25. Reding, K.W., et al., Adjuvant systemic therapy for BC in BRCA1/BRCA2 mutation carriers in a population-based study of risk of contralateral BC. BC Res Treat, 2010. 123(2): p. 491-8.

26. Phillips, K.A., et al., Tamoxifen and risk of contralateral BC for BRCA1 and BRCA2 mutation carriers. J Clin Oncol, 2013. 31(25): p. 3091-9.

27. Gronwald, J., et al., Duration of tamoxifen use and the risk of contralateral BC in BRCA1 and BRCA2 mutation carriers. BC Res Treat, 2014. 146(2): p. 421-7.

28. Swain, S.M., Tamoxifen and contralateral BC: the other side. J Natl Cancer Inst, 2001. 93(13): p. 9635.

29. Rutqvist, L.E., et al., Adjuvant tamoxifen therapy for early stage $B C$ and second primary malignancies. Stockholm BC Study Group. J Natl Cancer Inst, 1995. 87(9): p. 645-51.

30. Cook, L.S., et al., Population-based study of tamoxifen therapy and subsequent ovarian, endometrial, and BCs. J Natl Cancer Inst, 1995. 87(18): p. 1359-64.

31. Burstein, H.J., et al., Adjuvant endocrine therapy for women with hormone receptor-positive BC: american society of clinical oncology clinical practice guideline focused update. J Clin Oncol, 2014. 32(21): p. 2255-69.

32. Abderrahman, B. and V.C. Jordan, Long-term Adjuvant Tamoxifen Therapy and Decreases in Contralateral BC. JAMA Oncol, 2016.

33. Mellemkjaer, L., et al., Risk of contralateral BC after tamoxifen use among Danish women. Ann Epidemiol, 2014. 24(11): p. 843-8.

34. Ademuyiwa, F.O., et al., Managing BC in younger women: challenges and solutions. BC (Dove Med Press), 2016. 8: p. 1-12.

35. To, C., M.B. Sporn, and K.T. Liby, PARP inhibitors for chemoprevention-reply. Cancer Prev Res (Phila), 2014. 7(11): p. 1172.

\section{Figures}




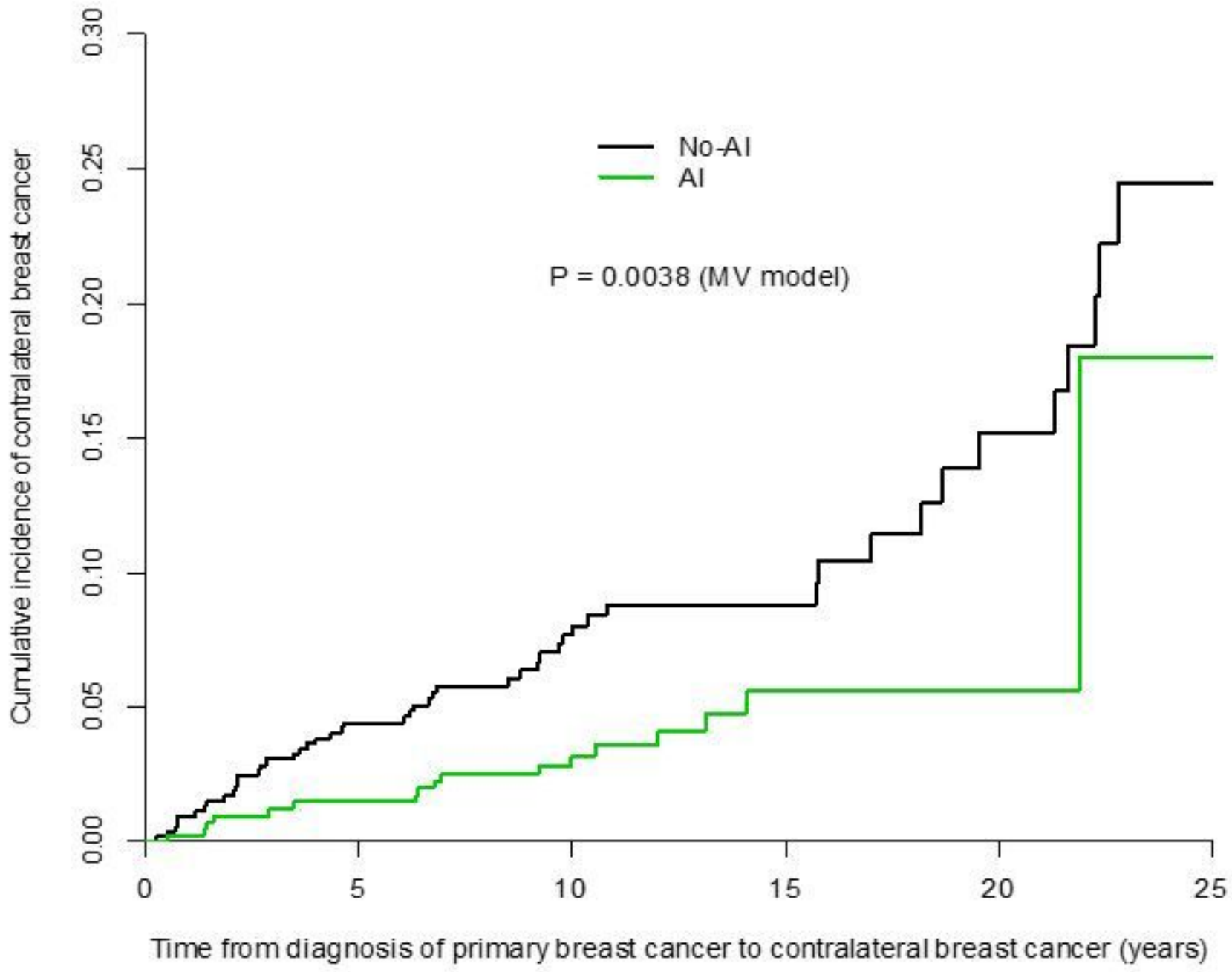

Figure 1

Cumulative incidence plot by adjuvant Al therapy 


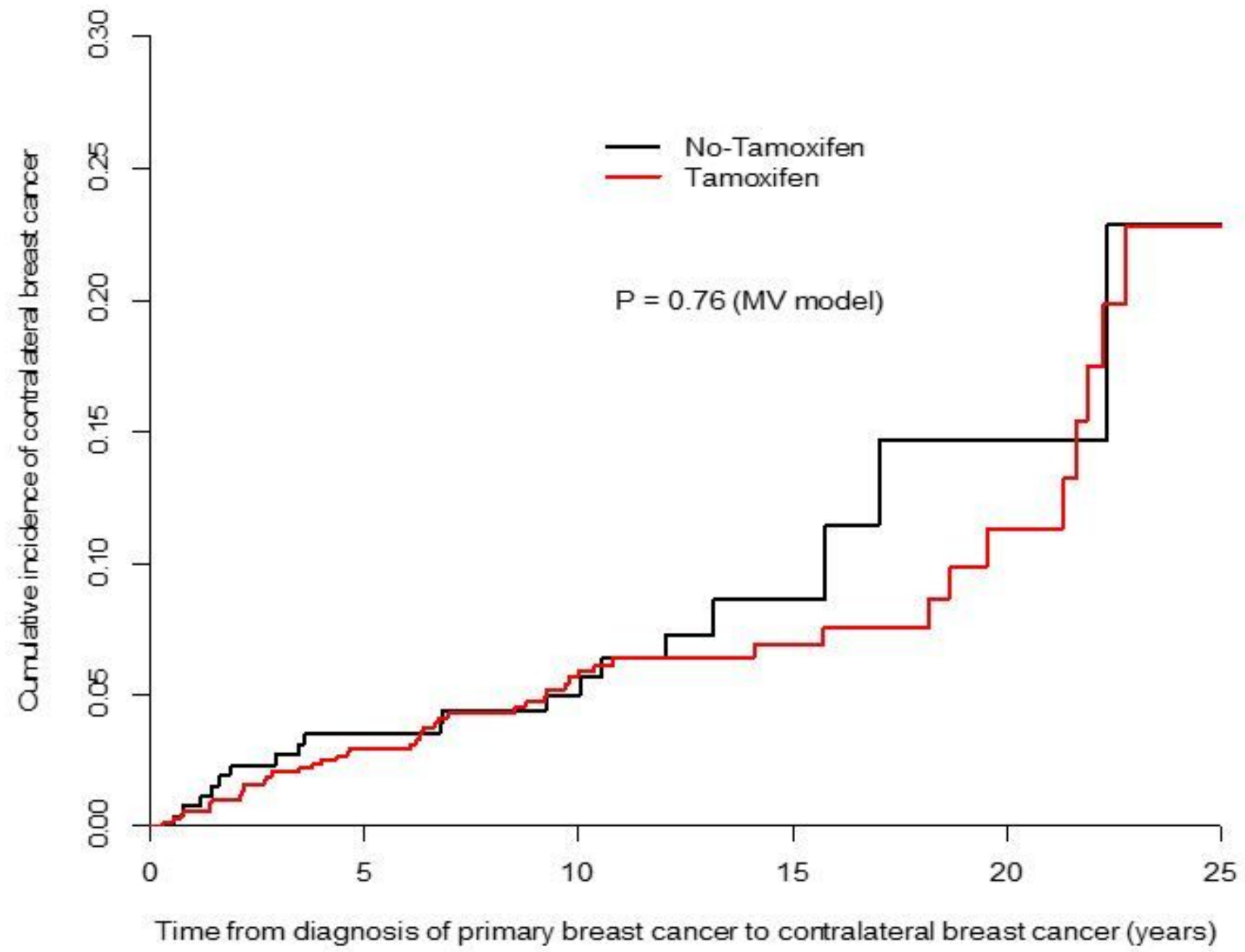

Figure 2

Cumulative incidence plot by adjuvant tamoxifen therapy 


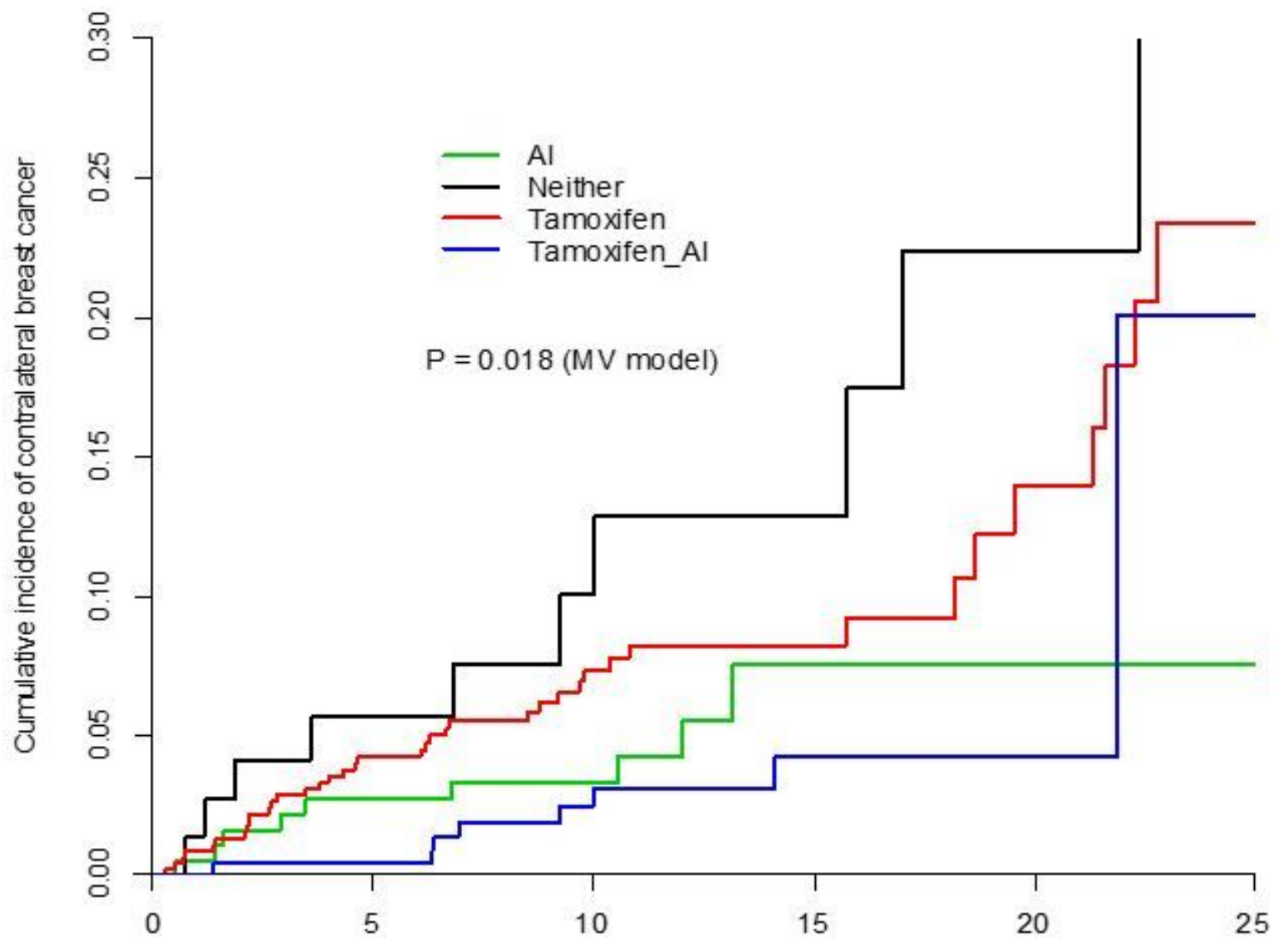

Time from diagnosis of primary breast cancer to contralateral breast cancer (years)

Figure 3

Cumulative incidence plot by adjuvant tamoxifen and/or Al therapy 


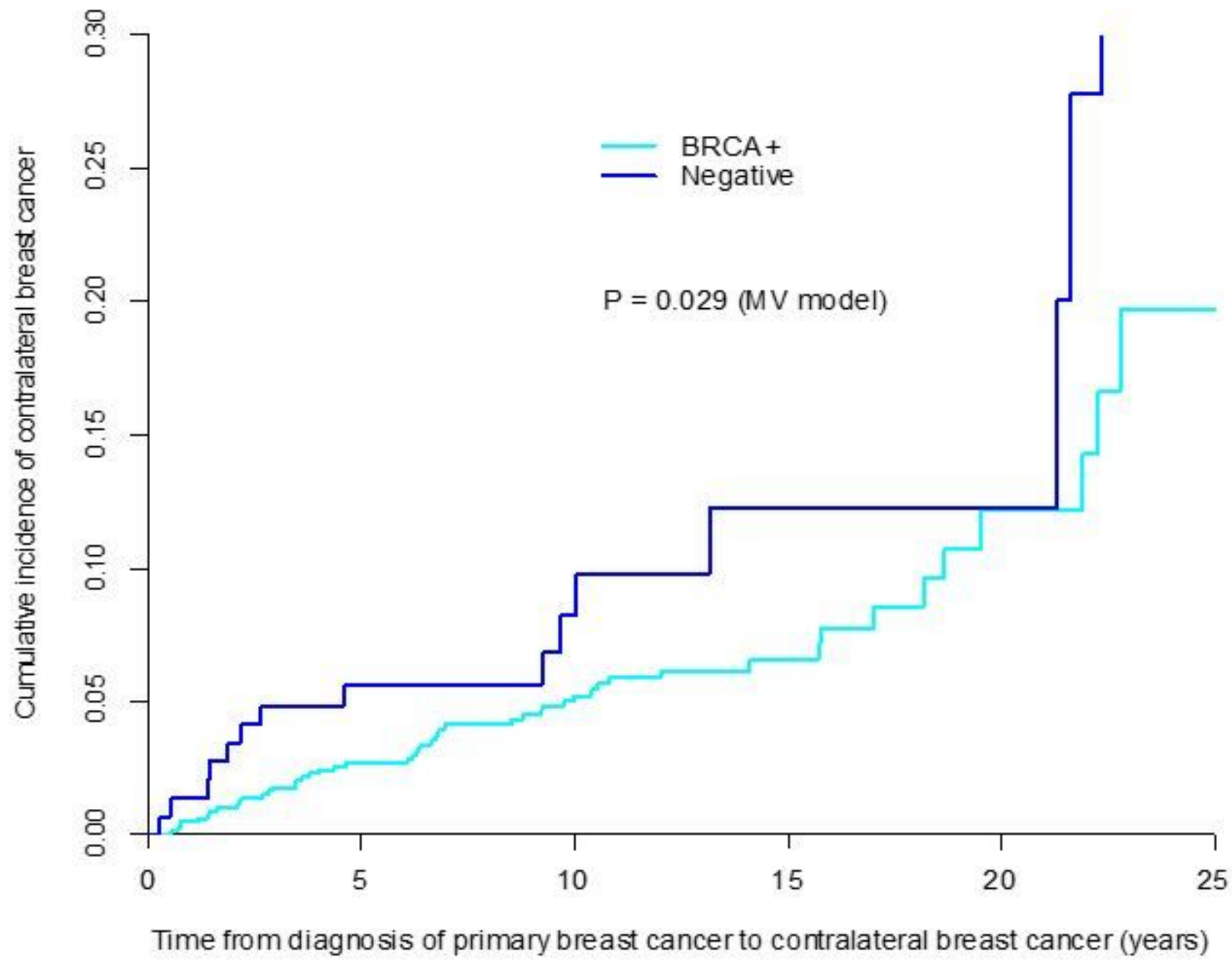

Figure 4

Cumulative incidence plot by BRCA status 


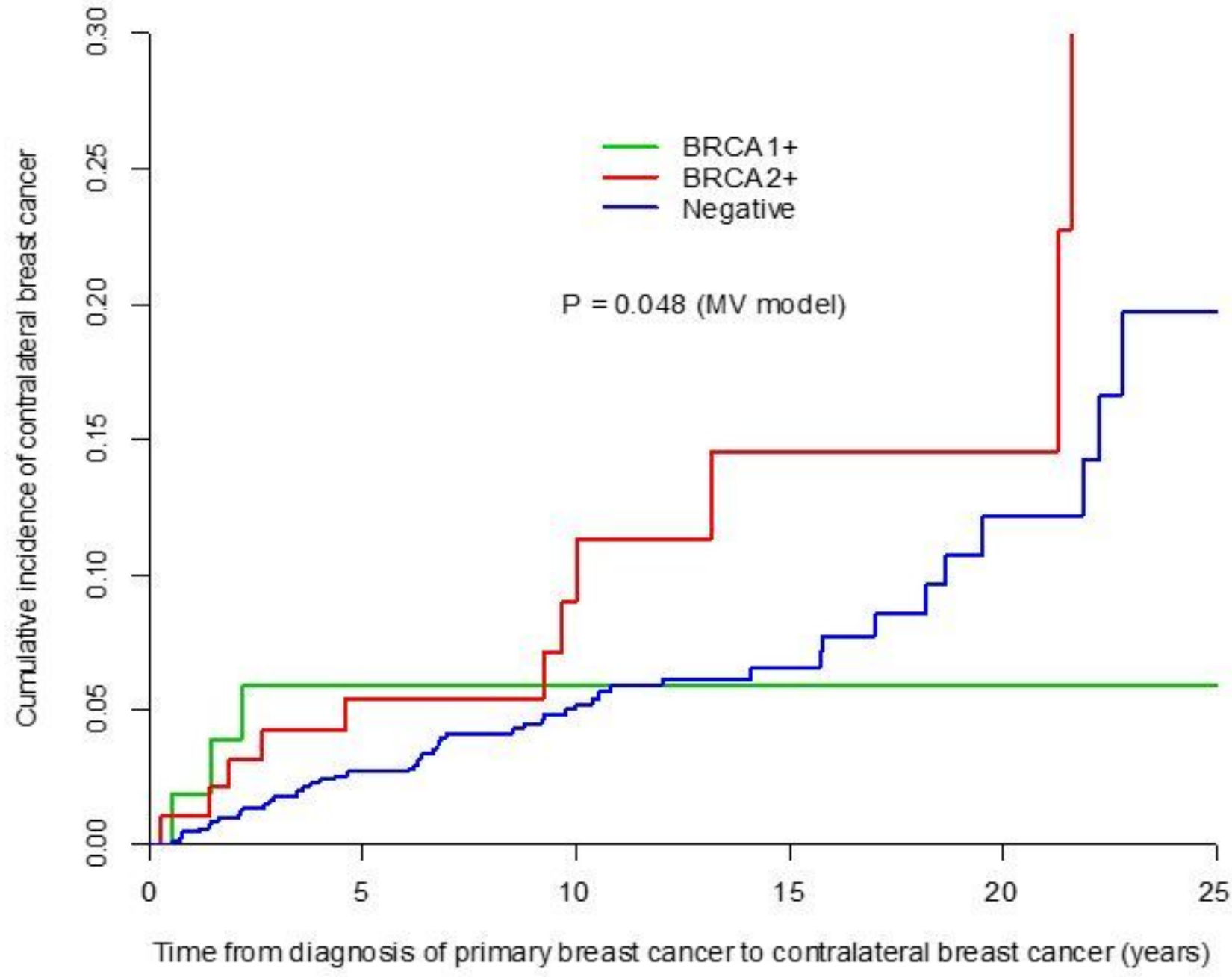

Figure 5

Cumulative incidence plot by BRCA status 


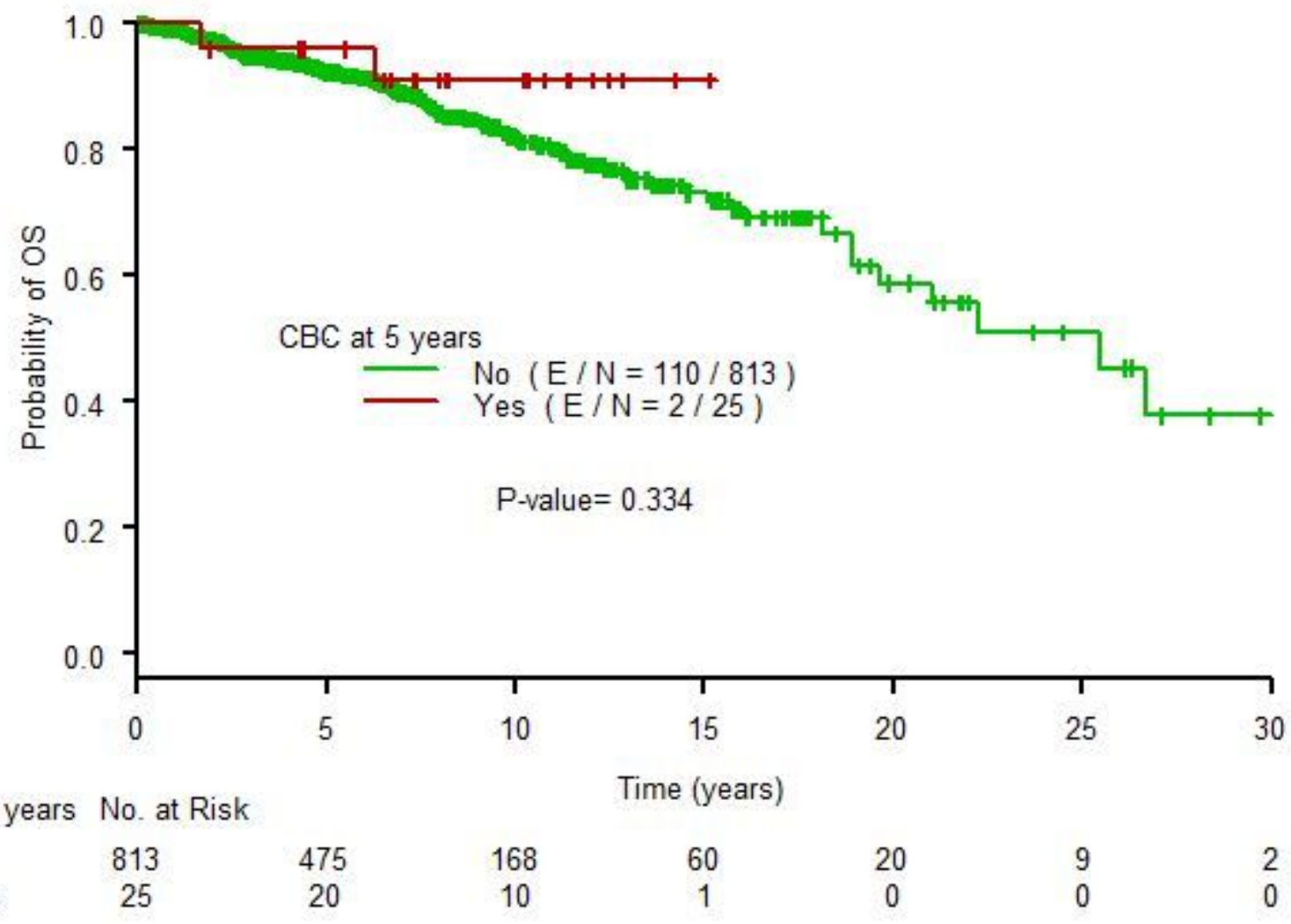

Figure 6

Kaplan-Meier curve of overall survival by CBC status 5 years post primary 


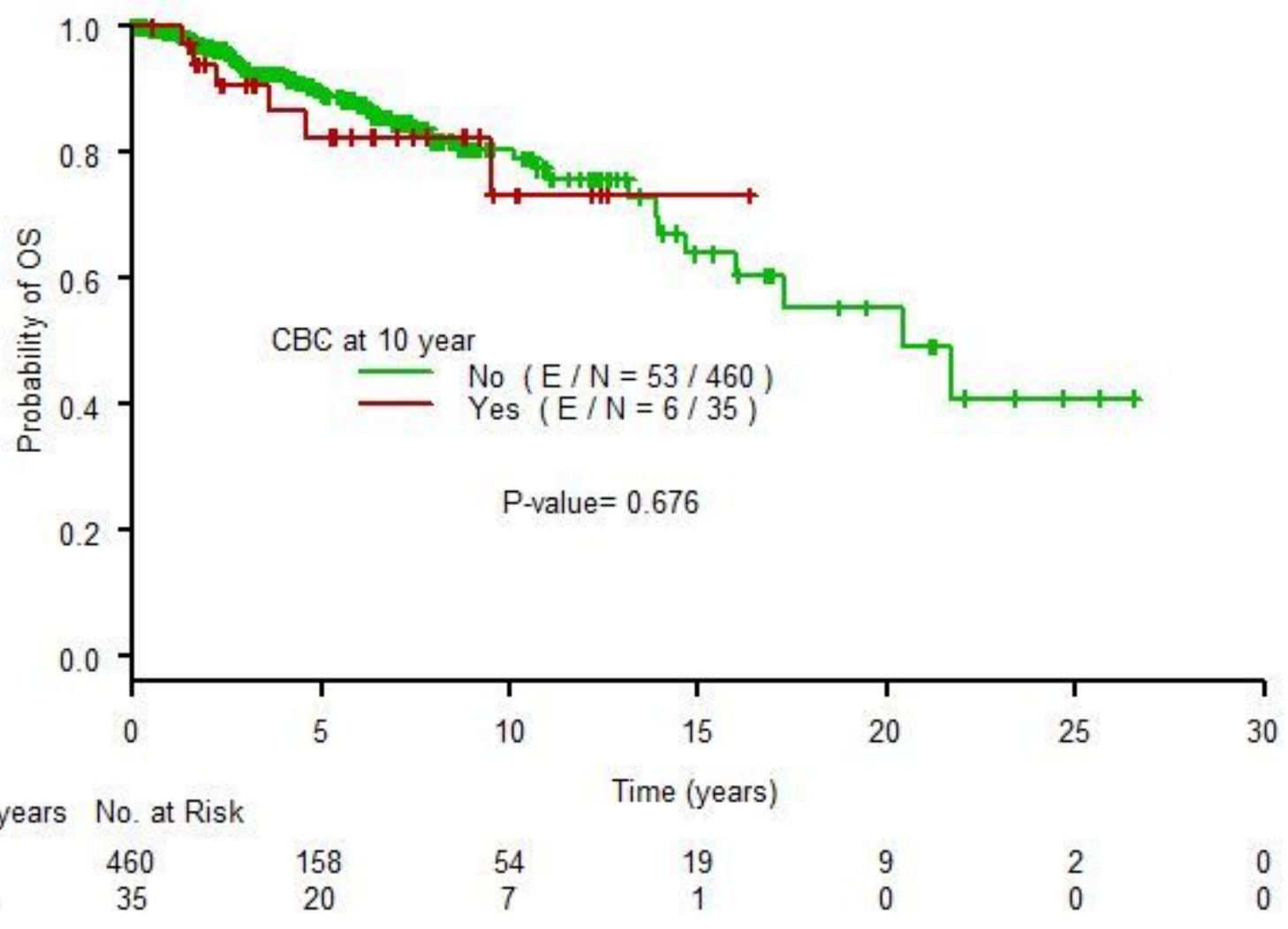

Figure 7

Kaplan-Meier curve of overall survival by $\mathrm{CBC}$ status 10 years post primary diagnosis

Figure 8

Kaplan-Meier curve of overall survival from primary diagnosis by BRCA status 